\title{
Guiding, bending, and splitting of electromagnetic waves in highly confined photonic crystal waveguides
}

\author{
Mehmet Bayindir* and E. Ozbay \\ Department of Physics, Bilkent University, Bilkent, 06533 Ankara, Turkey \\ B. Temelkuran \\ Department of Materials Science and Engineering \& Research Laboratory of Electronics, Massachusetts Institute of Technology, \\ Cambridge, Massachusetts 02139 \\ M. M. Sigalas \\ Communications and Optics Lab, Agilent Labs, Agilent Technologies, 3500 Deer Creek Road, MS26M9C, Palo Alto, California 94304 \\ C. M. Soukoulis, R. Biswas, and K. M. Ho \\ Ames Laboratory and Microelectronics Research Center, Iowa State University, Ames, Iowa 50011
}

(Received 18 October 2000; published 7 February 2001)

\begin{abstract}
We have experimentally demonstrated the guiding, bending, and splitting of electromagnetic (EM) waves in highly confined waveguides built around three-dimensional layer-by-layer photonic crystals by removing a single rod. Full transmission of the EM waves was observed for straight and bended waveguides. We also investigated the power splitter structures in which the input EM power could be efficiently divided into the output waveguide ports. The experimental results, dispersion relation and photon lifetime, were analyzed with a theory based on the tight-binding photon picture. Our results provide an important tool for designing photonic crystal based optoelectronic components.
\end{abstract}

DOI: 10.1103/PhysRevB.63.081107

PACS number(s): 42.70.Qs, 42.60.Da, 42.82.Et, 71.15.Ap

Photonic crystals, in which the propagation of electromagnetic waves is forbidden for a certain frequency range, ${ }^{1-3}$ provide a promising tool to control the flow of light in integrated optical devices. ${ }^{4,5}$ Therefore, there is a great deal of interest in developing photonic crystal-based waveguides where one can confine and efficiently guide the light around sharp corners. ${ }^{6-17}$ Guiding the light without losses, and even through sharp corners using twodimensional (2D) photonic crystals was proposed theoretically by Mekis et al. ${ }^{6}$ Later, researchers have reported experimental observation of waveguiding in 2D photonic crystals first at microwave, ${ }^{7}$ and then at optical frequencies. ${ }^{12-15}$ However, to avoid the leakage problem in 2D structures, either one has to extend the size of the photonic crystal in the vertical direction, or use a strong indexguiding mechanism in the vertical direction. ${ }^{15,17}$

A way to eliminate the leakage is to use a threedimensional (3D) photonic crystal. Recently, full confinement of the EM waves utilizing a 3D layer-by-layer photonic crystal structure has been theoretically studied. ${ }^{10,11}$ Although Noda et al. reported the fabrication of a 3D sharp bend waveguide at optical wavelengths, they have not reported any optical measurements on this sharp-bend structure. ${ }^{16}$

In this paper, we demonstrated that the guiding, bending, and splitting of EM waves could be achieved in highly confined waveguides which were constructed by removing a single rod from a perfect 3D layer-by-layer photonic crystal. The experimental results were in good agreement with a theory based on tight-binding (TB) approximation, $9,18,19$ and the simulation results of Chutinan and Noda. ${ }^{11}$

In our experiments, we used a layer-by-layer dielectric photonic crystal $^{20}$ based on square-shaped alumina rods
$(0.32 \mathrm{~cm} \times 0.32 \mathrm{~cm} \times 15.25 \mathrm{~cm})$, with center-to-center separation of $1.12 \mathrm{~cm}$. The crystal exhibits a threedimensional photonic band gap with a mid-gap frequency around $12 \mathrm{GHz}{ }^{21}$ Figure 1 shows the schematics of the measurement setup that was used in our experiments. We used an HP 8510C network analyzer and microwave horn antennas to measure the transmission-amplitude and the transmissionphase properties of a straight waveguide [Fig. 2(a)], a 90degree bended waveguide [Fig. 2(b)], and a power splitter structure [Fig. 2(c)]. We constructed the straight waveguide by removing a single rod from a single layer of a 6 unit cell (24 layer) photonic crystal, so that we had 12 layers at the top and 11 layers at the bottom of the removed rod. The polarization vector e of the incident EM wave was kept parallel to the stacking direction of the layers. We did not observe any guided modes for the other polarization in any of our experiments.

First, we tested the guiding of EM waves by measuring the transmission through a single missing rod. We observed full transmission of the EM waves for certain frequencies within the photonic stop band [Fig. 3(a)]. The full transmission within the waveguiding band was a proof of how well the wave was confined and guided without losses. The guiding band started from $11.37 \mathrm{GHz}$ and ended at $12.75 \mathrm{GHz}$. In Fig. 3(a), we also plotted the transmission spectra (dotted line) of the perfect crystal for comparison. The guiding was limited with the photonic band gap of the crystal, for which the crystal had the property of reflecting the EM waves in all directions.

In order to understand the underlying physics behind this single rod removed waveguide, we need to closely look at structure of the waveguide. Each vacancy just below the re- 


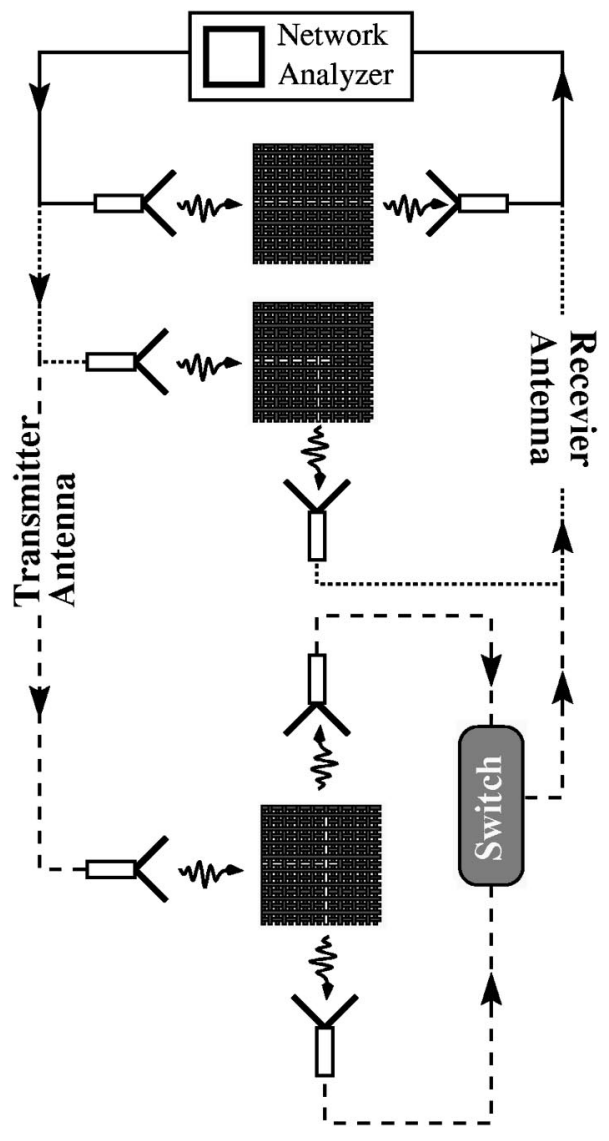

FIG. 1. Experimental setup for measuring the transmissionamplitude and transmission-phase spectra of the straight (solid) and bended (dotted) waveguides, and the power splitter structure (dashed).

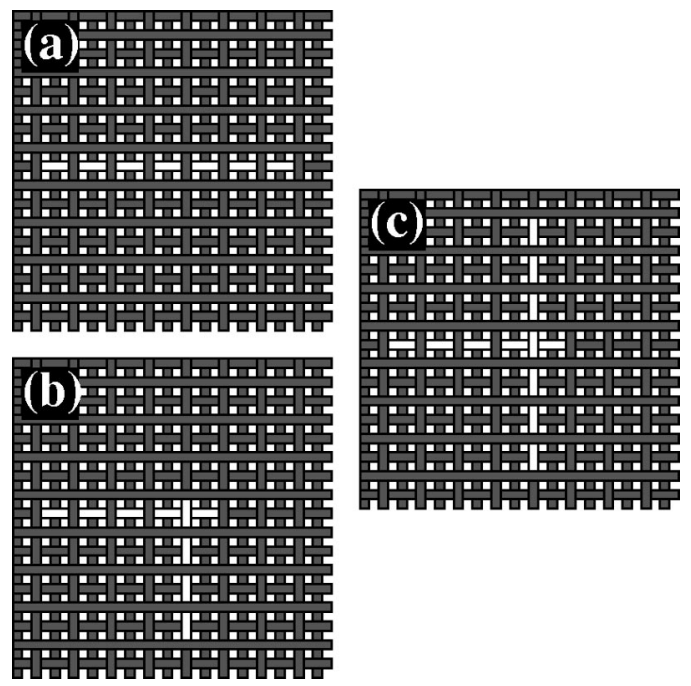

FIG. 2. (a) Schematic drawing of the straight waveguide was created by removing a single rod from one layer. (b) The 90-degree bended waveguide was obtained by removing two rods partially from two adjacent layers. (c) The power splitter structure was created by removing one rod partially from one layer and the whole rod from the adjacent upper layer.

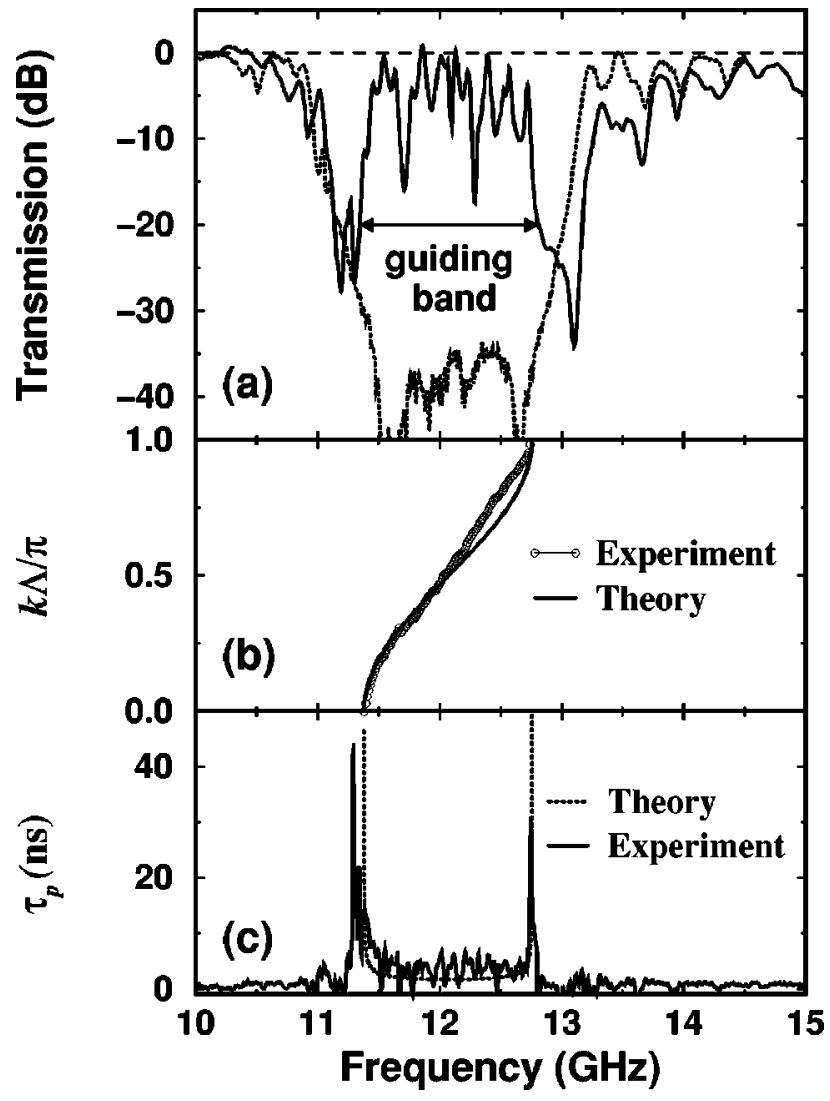

FIG. 3. (a) Transmission amplitude measured from a single rod removed waveguide structure (solid line). Nearly full transmission was achieved for certain frequencies. Transmission through a perfect crystal was plotted for comparison (dotted line). (b) The dispersion relation obtained from the transmission-phase measurements and the tight-binding approximation. (c) The measured and calculated delay time as a function of frequency. Photon lifetime $\left(\tau_{p}\right)$ increases drastically at the waveguiding band edges.

moved rod behaves as a boxlike cavity. The coupling between these localized cavity modes allow propagation of photons by hopping through the vacancy of the missing rod. Based on this observation, we can obtain the dispersion relation by measuring the transmission-phase characteristics and by using the TB approximation. Within the TB scheme, ${ }^{9}$ the dispersion relation is given by $\omega(k)=\Omega[1$ $+\kappa \cos (k \Lambda)]$. Here $\Omega=12.07 \mathrm{GHz}$ is the single defect frequency, $\Lambda=1.12 \mathrm{~cm}$ is the intercavity distance, $k$ is the wavevector, and $\kappa=-0.057$ is a TB parameter which was derived from the experimental data $(|\kappa|=\Delta \omega / 2 \Omega)$. As shown in Fig. 3(b), the measured dispersion relation is in good agreement with the TB prediction. We also measured and calculated the photon lifetime, i.e., delay time, of the single rod removed waveguide. Within the TB scheme, the photon lifetime can be written as $\tau_{p}(\omega)=\Lambda / v_{g}(\omega)$ $+2 \pi \Lambda / c{ }^{22}$ Here $v_{g}=\nabla_{k} \omega(k)$ is group velocity of the guided mode. Experimental and theoretical results show that the photon lifetime increases drastically, $\tau_{p} \rightarrow \infty$, at the waveguiding band edges [Fig. 3(c)].

We tested the bending of light through sharp corners in a waveguide structure shown in Fig. 2(b). This structure was 


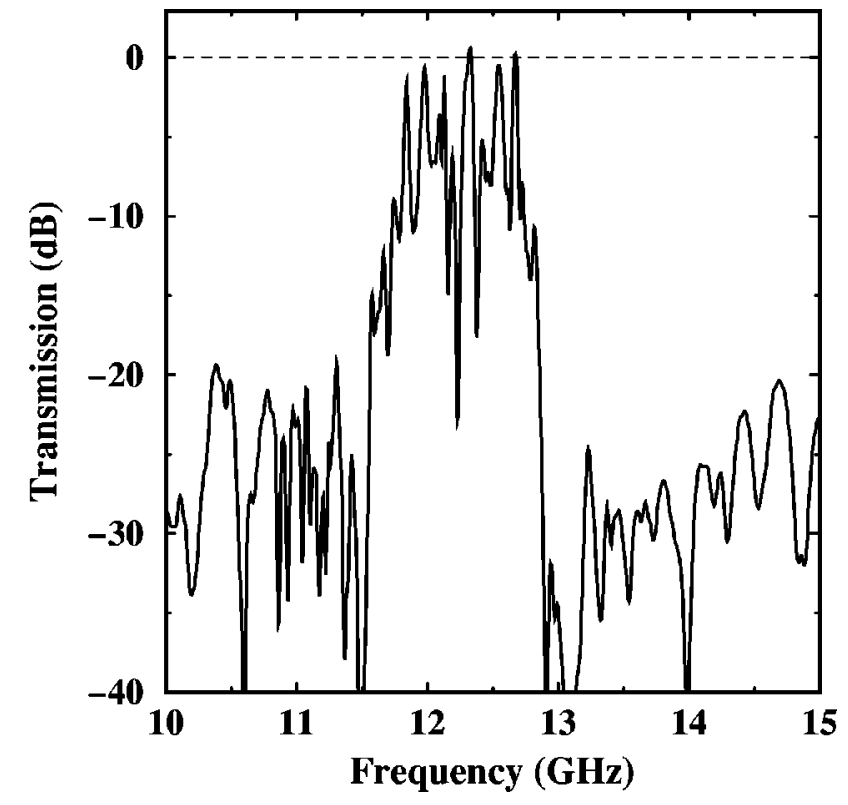

FIG. 4. Measured transmission characteristics of the 90-degree bended waveguide. A $100 \%$ transmission was obtained for certain frequencies throughout the guiding band.

constructed by removing part of a single rod from the $11^{\text {th }}$ layer, and part of another rod from the $12^{\text {th }}$ (adjacent) layer. The resulting vacancies of the missing parts of rods form a 90-degree sharp bend waveguide. The incident wave propagates along the first waveguide (missing portion of the rod on the $11^{\text {th }}$ layer), and successfully couples to the second waveguide on the $12^{\text {th }}$ layer, which is perpendicular to the propagation direction of the incident EM wave. As shown in Fig. 4, we observed a waveguiding band (extending from 11.55 to $12.87 \mathrm{GHz}$ ), for which the frequency range of the band was similar to the straight waveguide. The high transmission amplitudes reaching unity around certain frequencies showed that the EM waves were coupled and guided through the waveguide that contained a sharp bend. This observation is consistent with the TB picture, since the EM waves can propagate through coupled cavities without losses irrespective of change in the propagation direction.

At this point, we would like to compare our bended waveguide results with the simulations previously reported for this structure by Chutinan and Noda. ${ }^{11}$ Based on their simulation, the waveguiding band for sharp bend structure covers $67 \%$ of stop band of the photonic crystal which is very close to our experimental value of $68 \%$.

The power splitters ${ }^{23-26}$ are important for designing photonic crystal based optical components. To test the splitting idea, we removed one rod from the $12^{\text {th }}$ layer partially, and a

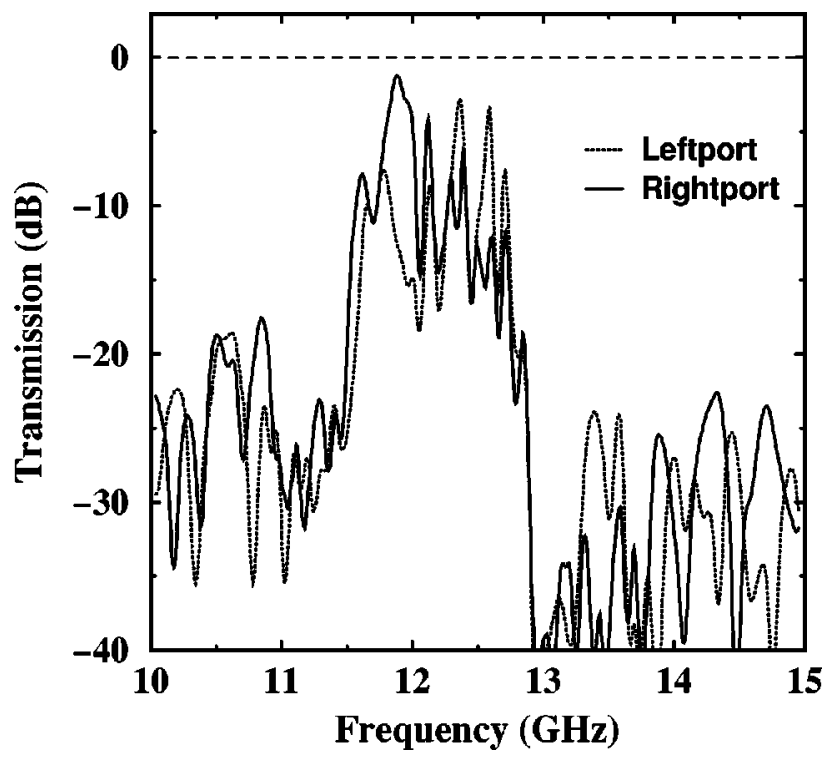

FIG. 5. Measured transmission spectra for the splitter structure illustrated in Fig. 2(c). The electromagnetic power in the input port splits into the two output ports throughout the guiding band.

whole rod from the $13^{\text {th }}$ layer with a crossed configuration [Fig. 2(c)]. Figure 5 shows transmission characteristics of this structure was measured. The EM wave inside the input waveguide channel was efficiently coupled to the output channels. Although we have achieved high transmission $(\sim 50 \%)$ for certain frequencies, the detected powers at each output port were mostly unequal throughout the waveguiding band.

In conclusion, we have experimentally demonstrated that a fully confined waveguide could be constructed by removing a single rod in a layer-by-layer photonic crystal. Full transmission of the EM waves was obtained through a straight waveguide and a waveguide with a sharp bend. Moreover, we have observed that the EM wave inside the input waveguide can be splitted into the output waveguide ports. Since the Maxwell's equations have no fundamental length scale, we expect that our microwave results can be extended to the optical frequencies. These results encourage the usage of the layer-by-layer photonic crystals in the design of future ultrasmall optoelectronic integrated circuits.

This work was supported by NATO Grant No. SfP971970, National Science Foundation Grant No. INT9820646, Turkish Department of Defense Grant No. KOBRA-001 and Thales JP8.04. Ames Laboratory is operated for the U.S. Department of Energy by Iowa State University under Contract No. W-7405-Eng-82.
*Author to whom correspondence should be addressed. Electronic address: bayindir@fen.bilkent.edu.tr

${ }^{1}$ J. D. Joannopoulos, R. D. Meade, and J. N. Winn, Photonic Crystals: Molding the Flow of Light (Princeton University Press, Princeton, NJ, 1995).

${ }^{2}$ For a recent review, see articles in Photonic Band Gap Materials, edited by C. M. Soukoulis (Kluwer, Dortrecht, 1996).
${ }^{3}$ J. D. Joannopoulos, P. R. Villeneuve, and S. Fan, Nature (London) 386, 143 (1997).

${ }^{4}$ H. Kosaka et al., Appl. Phys. Lett. 74, 1370 (1999).

${ }^{5}$ A. de Lustrac et al., Appl. Phys. Lett. 75, 1625 (1999).

${ }^{6}$ A. Mekis et al., Phys. Rev. Lett. 77, 3787 (1996).

${ }^{7}$ Shawn-Yu Lin, E. Chow, V. Hietala, P. R. Villeneuve, and J. D. Joannopoulos, Science 282, 274 (1998). 
${ }^{8}$ B. Temelkuran and E. Ozbay, Appl. Phys. Lett. 74, 486 (1999)

${ }^{9}$ Mehmet Bayindir, B. Temelkuran, and E. Ozbay, Phys. Rev. Lett. 84, 2140 (2000); Phys. Rev. B 61, R11 855 (2000).

${ }^{10}$ M. M. Sigalas et al., Microwave Opt. Technol. Lett. 23, 56 (1999).

${ }^{11}$ A. Chutinan and S. Noda, Appl. Phys. Lett. 75, 3739 (1999).

${ }^{12}$ T. Baba, N. Fukaya, and J. Yonekura, Electron. Lett. 35, 654 (1999).

${ }^{13}$ M. Tokushima, H. Kosaka, A. Tomita, and H. Yamada, Appl. Phys. Lett. 76, 952 (2000).

${ }^{14}$ M. Loncar et al., Appl. Phys. Lett. 77, 1937 (2000).

${ }^{15}$ S. Y. Lin, E. Chow, S. G. Johnson, and J. D. Joannopoulos, Opt. Lett. 25, 1297 (2000).

${ }^{16}$ S. Noda, K. Tomoda, N. Yamamoto, and A. Chutinan, Science 289, 604 (2000).

${ }^{17}$ S. G. Johnson, P. R. Villeneuve, S. Fan, and J. D. Joannopoulos, Phys. Rev. B 62, 8212 (2000).
${ }^{18}$ N. W. Ashcroft and N. D. Mermin, Solid State Physics (Sounders, Philadelphia, 1976) p. 175.

${ }^{19}$ N. Stefanou and A. Modinos, Phys. Rev. B 57, 12127 (1998); C. Martijn de Sterke, Phys. Rev. E 57, 3502 (1998); E. Lidorikis, M. M. Sigalas, E. N. Economou, and C. M. Soukoulis, Phys. Rev. Lett. 81, 1405 (1998); A. Yariv, Y. Xu, R. K. Lee, and A. Scherer, Opt. Lett. 24, 711 (1999).

${ }^{20}$ K. M. Ho, C. T. Chan, C. M. Soukoulis, R. Biswas, and M. M. Sigalas, Solid State Commun. 89, 413 (1994).

${ }^{21}$ E. Ozbay, J. Opt. Soc. Am. B 13, 1945 (1996).

${ }^{22}$ Mehmet Bayindir and E. Ozbay, Phys. Rev. B 62, R2247 (2000).

${ }^{23}$ Mehmet Bayindir, B. Temelkuran, and E. Ozbay, Appl. Phys. Lett. 77, 3902 (2000).

${ }^{24}$ J. Yonekura, M. Ikeda, and T. Baba, J. Lightwave Technol. 17, 1500 (1999).

${ }^{25}$ R. W. Ziolkowski and M. Tanaka, Opt. Quantum Electron. 31, 843 (1999).

${ }^{26}$ T. Sondergaard and K. H. Dridi, Phys. Rev. B 61, 15688 (2000). 